University of Nebraska - Lincoln

DigitalCommons@University of Nebraska - Lincoln

1982

\title{
Variability in Nest Survival Rates and Implications to Nesting Studies
}

\author{
A. T. Klett \\ USGS Northern Prairie Wildlife Research Center \\ Douglas H. Johnson \\ USGS Northern Prairie Wildlife Research Center, Douglas_H_Johnson@usgs.gov
}

Follow this and additional works at: https://digitalcommons.unl.edu/usgsnpwrc

Part of the Other International and Area Studies Commons

Klett, A. T. and Johnson, Douglas H., "Variability in Nest Survival Rates and Implications to Nesting Studies" (1982). USGS Northern Prairie Wildlife Research Center. 191.

https://digitalcommons.unl.edu/usgsnpwrc/191

This Article is brought to you for free and open access by the US Geological Survey at DigitalCommons@University of Nebraska - Lincoln. It has been accepted for inclusion in USGS Northern Prairie Wildlife Research Center by an authorized administrator of DigitalCommons@University of Nebraska - Lincoln. 


\title{
VARIABILITY IN NEST SURVIVAL RATES AND IMPLICATIONS TO NESTING STUDIES
}

\author{
A. T. Klett and Douglas H. Johnson \\ Northern Prairie Wildlife Research Center, Jamestown, North Dakota 58401 USA
}

\begin{abstract}
We used four reasonably large samples (83-213) of Mallard (Anas platyrhynchos) and Blue-winged Teal ( $A$. discors) nests on an interstate highway right-of-way in southcentral North Dakota to evaluate potential biases in hatch-rate estimates. Twelve consecutive, weekly searches for nests were conducted with a cable-chain drag in 1976 and 1977. Nests were revisited at weekly intervals. Four methods were used to estimate hatch rates for the four data sets: the Traditional Method, the Mayfield Method, and two modifications of the Mayfield Method that are sometimes appropriate when daily mortality rates of nests are not constant. Hatch rates and the average age of nests at discovery declined as the interval between searches decreased, suggesting that mortality rates were not constant in our samples. An analysis of variance indicated that daily mortality rates varied with the age of nests in all four samples. Mortality was generally highest during the early laying period, moderately high during the late laying period, and lowest during incubation. We speculate that this relationship of mortality to nest age might be due to the presence of hens at nests or to differences in the vulnerability of nest sites to predation. A modification of the Mayfield Method that accounts for age-related variation in nest mortality was most appropriate for our samples. We suggest methods for conducting nesting studies and estimating nest success for species possessing similar nesting habits. Received 21 January 1981, accepted 1 July 1981.
\end{abstract}

INVESTIGATORS have attempted to determine the nesting success of birds for many purposes and have used diverse methods as they studied species with different breeding strategies in a wide range of habitats. One characteristic common to most studies is the method used to calculate hatch rates: the percentage of nests in an observed sample in which at least one egg hatched (referred to as the Traditional Method in this paper). Several investigators (Lack 1954: 74, Coulson 1956, Hammond and Forward 1956, Peakall 1960) recognized that hatch rates calculated in this way were biased. Hammond and Forward (1956) warned, "Neglect of consideration for the length of time nests are under observation as compared to the total period they are exposed to predation would lead to a recorded success higher than that actually occurring." Eggs found in older nests are more likely to hatch than those found in younger nests, because they have already survived a period of risk to which the latter have yet to be exposed. By following this reasoning to the ultimate, it is clear that only samples of nests found on the day they are initiated will yield an unbiased hatch rate. The bias will increase with the average age of nests at discovery.
Mayfield (1961) proposed a method that accounts for bias related to the age of nests at discovery by introducing into the calculations the period of time nests are under observation. Mayfield's Method will greatly reduce the bias inherent in the hatch rate calculated by the Traditional Method if daily mortality rates are reasonably constant for all nests in the sampled population.

Some progress has also been made in analyzing nesting data for which daily mortality rates are not constant. Mayfield (1961, 1975) and Johnson (1979) described a method of calculating nest success when daily mortality varies among laying, incubation, and nestling stages. Johnson (1979) also proposed a method to use when the daily mortality of nests varies between population components such as yearling and older nesters. These methods will be described later in this paper.

The researchers who developed these methods often depended upon hypothetical population models or pooled samples from studies conducted over a period of years and sometimes in different areas. Our study is an attempt to evaluate these methods by applying them to four reasonably large samples of nests 
of two species, Mallard (Anas platyrhynchos) and Blue-winged Teal ( $A$. discors), that were observed during 2 yr on a single study area.

The specific purposes of this paper are (1) to evaluate the variation in daily mortality rates in four samples of nests, (2) to determine the importance of variation in estimating hatch rates by the methods listed above, (3) to discuss the appropriate methodologies for evaluating our data, and (4) to recommend methods to researchers studying species with similar characteristics.

\section{StUdY AREA}

Nest data were collected in 1976 and 1977 on 37 $\mathrm{km}$ of right-of-way along Interstate 94 in Stutsman and Kidder counties, North Dakota. The average width of the cover strip on each side of the highway was $34 \mathrm{~m}$; the total area searched was about 250 ha. The right-of-way traversed an area typified by numerous small wetlands and scattered shallow marshes and lakes. Water levels in the wetlands were high in the spring of 1976, but during the summer of 1976 and throughout the 1977 nesting season all of the temporary and most of the seasonal wetlands (classified according to Stewart and Kantrud 1971) were dry. The vegetation on the right-of-way consisted of introduced grasses and legumes dominated by smooth brome (Bromus inermis), except on drier sites, where crested wheatgrass (Agropyron cristatum) was dominant (Voorhees and Cassel 1980). Land use adjacent to the study area was chiefly small grain cropland, tame hayland, and native prairie pasture. Potential egg predators observed on or near the study area were the red fox (Vulpes vulpes), striped skunk (Mephitis mephitis), mink (Mustela vison), long-tailed weasel (M. frenata), badger (Taxidea taxus), raccoon (Procyon lotor), Franklin's ground squirrel (Citellus franklinii), and gulls (Larus spp.).

\section{Methods AND Results}

Gathering the samples.-We conducted 12 consecutive weekly searches for nests in both 1976 and 1977 by flushing nesting ducks with a 53-m-long cable-chain drag (Higgins et al. 1969) towed between two vehicles. The searches were initiated in mid-April, and each was completed in 3-5 mornings. The date found, number of eggs, and their incubation stage (Weller 1956) were used to estimate the initiation date and the expected hatch date. A "nest" was defined as any clutch found with one or more viable eggs. We assumed that one egg was laid each day, incubation began on the day the last egg was laid, and incubation periods were 25 days for Mallards and 23 days for Blue-winged Teal. We revisited nests during subsequent searches and recorded additional data on nest status, presence of the hen, and flushing response to the cable-chain drag. Hen presence, egg temperature, apparent nest age, appearance of egg fragments, and other evidence were used to determine nest status (still viable or eggs were hatched, destroyed or abandoned). Of 563 clutches that were found, 22 failed because of nest searching operations or other human activities, 9 were abandoned, 263 were destroyed by predators, and 269 hatched. Only clutches that hatched or were destroyed by predators are considered in this paper (Table 1).

The Mayfield Method of calculating hatch rates.-Mayfield's (1961) Method for calculating hatch rates incorporates the period of time a nest was under observation and vulnerable to loss. He calculated a daily mortality rate $(m)$ by dividing the number of clutches that failed to hatch by the total number of days all nests were under observation and exposed to loss (exposure days, Table 1). If the date a nesting attempt failed was unknown, he used as the exposure period half the interval between the last two visits plus the known survival period. [Miller and Johnson (1978) suggested that only $40 \%$ of the interval during which a nesting attempt failed be added to the known exposure when that interval was long. We used $50 \%$ of the interval in this study because nests were visited approximately every 7 days.] The hatch rate was derived by raising the daily survival rate $(s=1-m)$ to a power equal to the average life-span (laying + incubation) of successful clutches. For comparative purposes, we assumed this period to be 34 days for both species [see Mayfield $(1961,1975)$ for more complete details on the computations]. This method is based on the assumption that all nests are subject to the same constant daily mortality rate from the day the first egg is laid until the nest is terminated by success or failure. Deviations from this assumption can result in biased estimators; Johnson (1979), however, considered this bias insignificant with as much as a twofold difference in daily mortality rates between subpopulations of nests.

The number of nests found and hatch rates differed sharply between the $2 \mathrm{yr}$ (Table 1 ). More nests were found in 1976 when wetland conditions were favorable, but hatch rates 
TABLE 1. Basic nest information and hatch rates.

\begin{tabular}{|c|c|c|c|c|}
\hline & \multicolumn{2}{|c|}{1976} & \multicolumn{2}{|c|}{1977} \\
\hline & Mallard & $\begin{array}{c}\text { Blue-winged } \\
\text { Teal }\end{array}$ & Mallard & $\begin{array}{c}\text { Blue-winged } \\
\text { Teal }\end{array}$ \\
\hline \multicolumn{5}{|l|}{ Number of nests } \\
\hline $\begin{array}{l}\text { Destroyed } \\
\text { Hatched }\end{array}$ & $\begin{array}{l}89 \\
41\end{array}$ & $\begin{array}{r}118 \\
95\end{array}$ & $\begin{array}{l}28 \\
55\end{array}$ & $\begin{array}{l}28 \\
78\end{array}$ \\
\hline Exposure days & 1,966 & 3,460 & 1,651 & 2,140 \\
\hline \multicolumn{5}{|l|}{ Hatch rates } \\
\hline $\begin{array}{l}\text { Traditional Method } \\
\text { Mayfield Method } \\
\text { Product Method } \\
\text { Intercept Method }\end{array}$ & $\begin{array}{r}31.5 \\
20.7 \\
17.3 \\
7.7\end{array}$ & $\begin{array}{l}44.6 \\
30.7 \\
29.5 \\
20.0\end{array}$ & $\begin{array}{l}66.3 \\
55.9 \\
43.2 \\
13.5\end{array}$ & $\begin{array}{l}73.6 \\
63.9 \\
51.1 \\
22.2\end{array}$ \\
\hline
\end{tabular}

were higher in 1977. We suspect that red fox, Franklin's ground squirrel, and striped skunk were important egg predators in both years but that less predation by red fox occurred in 1977 . Characteristically, hatch rates calculated by the Mayfield Method were lower than those calculated by the Traditional Method for both species in both years. Although not evident from Table 1, such discrepancies would diminish with increases in the true hatch rate and would vanish if all nesting attempts were successful.

Effect of sampling pattern on estimates of hatch rates.-We explored the effect of sampling intensity on hatch-rate estimates by simulating various search patterns from the 1976 Mallard data. Table 2 shows the results of searches made at intervals of 1 week, 2 weeks, 3 weeks, and 4 weeks. The 12 searches conducted at weekly intervals provided the basic data. There are 2 possible search patterns of 6 searches each when searches are conducted at 2-week intervals (weeks $1,3,5,7,9$, and 11 ; weeks $2,4,6$, 8,10 , and 12), 3 patterns of 4 searches each for 3 -week intervals, and 4 patterns of 3 searches each for 4-week intervals. The number of nests, their average age at discovery, and hatch rates were determined for each search pattern. Values in Table 2 are averages from all possible search patterns in the designated interval.

As expected, more nests are found with more intensive sampling; of greater interest is the decline in hatch rates and average age of nests at discovery when search intervals are shortened. The age of nests found during searches made with a 4-week interval is nearly twice that of the nests discovered with weekly searches. Hatch rates calculated by both the Mayfield and Traditional Methods become in- creasingly biased with less frequent searches. The Mayfield Method substantially reduces this bias, but, nonetheless, these results imply that the hatch-rate estimate would decline with additional sampling; apparently daily mortality rates are not constant in this sample. Similar trends were noted in the other data sets.

Variation related to age of nest and date.-We sought to estimate daily mortality rates according to age of nest and calendar period and to determine if the variation in daily mortality rates was related to these variables. We determined daily mortality rates for nests in each 5day age class (1-5 days old, 6-10 days old, etc.) and for each 10-day calendar period (days 110$119=19-28$ April; $120-129=29$ April-8 May; etc.). (To avoid confusion, the terms AGE and DATE will appear in capital letters when they refer to the 5-day age classes and 10-day calendar periods.) We calculated the probability of nest losses and exposure days occurring in each category and then determined the daily mortality rates by dividing (see Appendix for details). We followed the latter procedure using the Mallard and Blue-winged Teal data sets in each year and tabulated the results by AGE and by DATE. For example, the overall daily

TABLE 2. A relationship of hatch rates and average nest age to sampling intensity from 1976 Mallard data.

\begin{tabular}{|c|c|c|c|c|}
\hline \multirow{2}{*}{$\begin{array}{c}\text { Search } \\
\text { interval } \\
\text { in } \\
\text { weeks }\end{array}$} & \multirow{2}{*}{$\begin{array}{l}\text { Number } \\
\text { of nests } \\
\text { found }\end{array}$} & \multirow{2}{*}{$\begin{array}{c}\text { Average } \\
\text { age at } \\
\text { discovery } \\
\text { (days) }\end{array}$} & \multicolumn{2}{|c|}{ Hatch rates } \\
\hline & & & Traditional & Mayfield \\
\hline 1 & 130 & 8.9 & 31.5 & 20.7 \\
\hline 2 & 97 & 11.6 & 37.8 & 23.6 \\
\hline 3 & 80 & 13.8 & 41.6 & 24.2 \\
\hline 4 & 68 & 15.5 & 45.8 & 25.4 \\
\hline
\end{tabular}


TABLE 3. Exposure, losses, and estimated daily mortality rate $(m)$ for 1976 Blue-winged Teal nests according to age of nest and calendar period.

\begin{tabular}{|c|c|c|c|c|c|c|c|c|}
\hline \multirow{2}{*}{$\begin{array}{l}\text { Calendar } \\
\text { period }\end{array}$} & \multicolumn{7}{|c|}{ Age of nest (days) } & \multirow[b]{2}{*}{ Total } \\
\hline & $1-5$ & $6-10$ & $11-15$ & $16-20$ & $21-25$ & $26-30$ & $\geqslant 31$ & \\
\hline \multicolumn{9}{|l|}{$120-129$} \\
\hline Exposure & 17 & 13 & 2 & 0 & 0 & 0 & 0 & 32 \\
\hline Losses & 0 & 0 & 0 & 0 & 0 & 0 & 0 & 0 \\
\hline$m$ & 0 & 0 & 0 & - & - & - & - & 0 \\
\hline \multicolumn{9}{|l|}{$130-139$} \\
\hline Exposure & 41.314 & 96.736 & 107.929 & 27.202 & 2.328 & 0 & 0 & 275.509 \\
\hline Losses & 1.883 & 2.569 & 5.381 & 2.436 & 0.115 & 0 & 0 & 12.384 \\
\hline$m$ & 0.0456 & 0.0266 & 0.0499 & 0.0896 & 0.0494 & - & - & 0.0449 \\
\hline \multicolumn{9}{|l|}{$140-149$} \\
\hline Exposure & 40.888 & 191.551 & 282.710 & 212.401 & 84.222 & 15.104 & 2.000 & 828.876 \\
\hline Losses & 2.201 & 7.198 & 9.444 & 6.046 & 3.295 & 0.104 & 0 & 28.288 \\
\hline$m$ & 0.0538 & 0.0376 & 0.0334 & 0.0285 & 0.0391 & 0.0069 & 0 & 0.0341 \\
\hline \multicolumn{9}{|l|}{$150-159$} \\
\hline Exposure & 11.848 & 63.653 & 208.746 & 307.019 & 279.517 & 171.235 & 43.540 & $1,085.558$ \\
\hline Losses & 0.348 & 1.878 & 9.072 & 8.243 & 6.445 & 4.077 & 2.730 & 32.793 \\
\hline$m$ & 0.0294 & 0.0295 & 0.0435 & 0.0268 & 0.0231 & 0.0238 & 0.0627 & 0.0302 \\
\hline \multicolumn{9}{|l|}{$160-169$} \\
\hline Exposure & 9.856 & 37.899 & 77.037 & 112.356 & 185.893 & 263.282 & 184.725 & 871.048 \\
\hline Losses & 1.269 & 2.530 & 2.608 & 5.185 & 5.783 & 6.128 & 4.410 & 27.913 \\
\hline$m$ & 0.1288 & 0.0668 & 0.0339 & 0.0461 & 0.0311 & 0.0233 & 0.239 & 0.0320 \\
\hline \multicolumn{9}{|l|}{$170-179$} \\
\hline Exposure & 0 & 12.054 & 33.727 & 33.844 & 57.980 & 81.498 & 77.090 & 296.193 \\
\hline Losses & 0 & 1.663 & 1.320 & 1.610 & 1.729 & 2.922 & 1.924 & 11.168 \\
\hline$m$ & - & 0.1380 & 0.0391 & 0.0476 & 0.0298 & 0.359 & 0.0250 & 0.0377 \\
\hline \multicolumn{9}{|l|}{$180-189$} \\
\hline Exposure & 0 & 2.443 & 1.908 & 14.599 & 23.413 & 35.009 & 32.578 & 109.950 \\
\hline Losses & 0 & 0.538 & 0.462 & 0.385 & 1.125 & 1.750 & 1.185 & 5.445 \\
\hline$m$ & - & 0.2202 & 0.2421 & 0.0264 & 0.0481 & 0.0500 & 0.0364 & 0.0495 \\
\hline \multicolumn{9}{|l|}{ Total } \\
\hline Exposure & 120.906 & 417.336 & 714.057 & 707.421 & 633.353 & 566.128 & 339.933 & $3,499.134$ \\
\hline Losses & 5.701 & 16.376 & 28.287 & 23.905 & 18.492 & 14.981 & 10.249 & 117.991 \\
\hline$m$ & 0.0472 & 0.0392 & 0.0396 & 0.0338 & 0.0292 & 0.0265 & 0.0302 & 0.0337 \\
\hline
\end{tabular}

mortality rate for the 1976 Blue-winged Teal data is 0.0337 , derived from 118 nests lost during 3,499.134 nest-days of exposure (Table 3). Daily mortality rates by AGE and DATE are based on expected exposure and differ slightly from those presented in Table 1.

A graph of daily mortality rate by age of nest suggests that the rate is not constant, but declines among older nests (Fig. 1). Except for Blue-winged Teal in 1976, the decline is pronounced through the 6-10-day age class, after which the mortality rate becomes fairly constant. Nests from all dates have been combined in this graph, and any effect of DATE would be confounded with the AGE effect illustrated.

The graph of mortality rates plotted by DATE indicates no consistent trends (Fig. 2). The Mallard and Blue-winged Teal data sets in 1976 display a concave curve, higher rates early and late in the season and lower rates in the mid- dle; the low rate for the Mallard during the period 110-119 was calculated from a very small sample. In 1977 Blue-winged Teal mortality rates decreased slightly throughout the season, and rates for the Mallard were fairly constant. This graph is confounded by AGE effects. For example, nests found early in the season tend to be "younger" than those found later, and we suspect from Fig. 1 that younger nests tend to have higher mortality rates.

We used analysis of variance techniques to sort out the relationship of daily mortality rates to age of nest and calendar period. [The program used was the GLM procedure of SAS76 (Barr et al. 1976).] The dependent variable was the estimated daily mortality rate within an AGE-DATE category. Data were weighted by the exposure in a category to account for differing sample sizes among categories. To each of the four data sets we fitted a variety of 


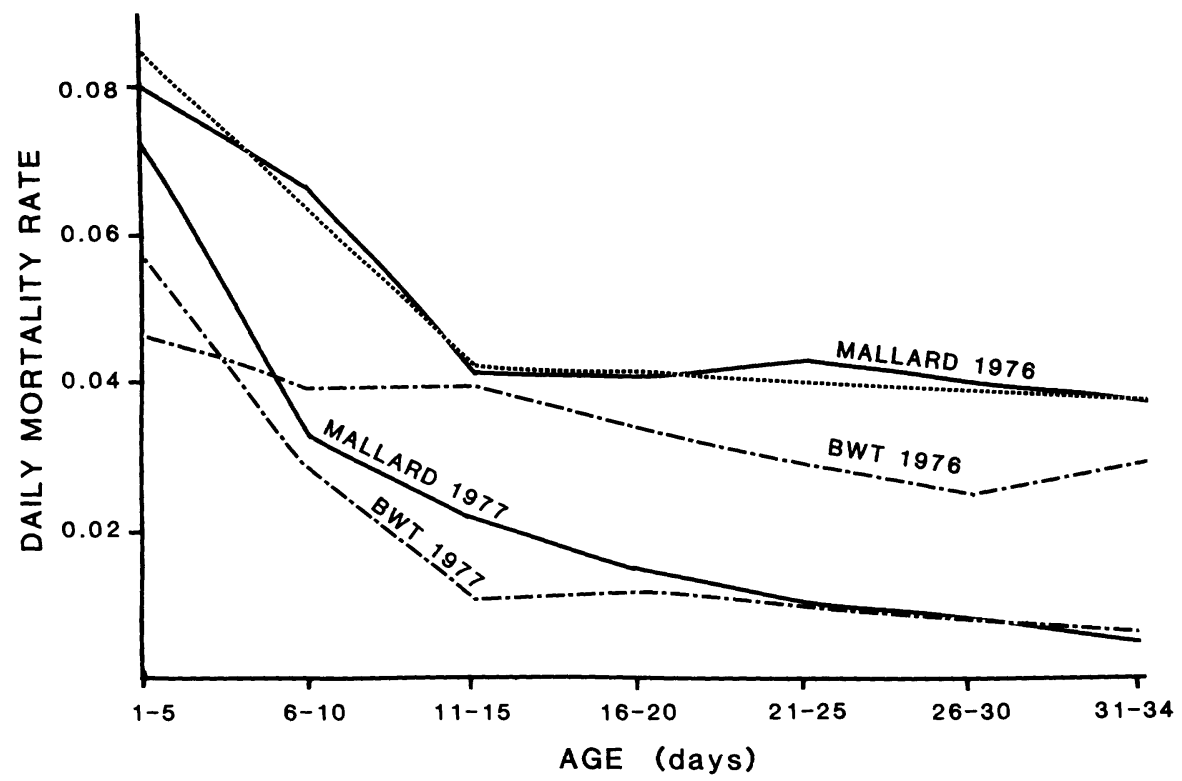

Fig. 1. Daily mortality of nests in relation to the number of days since initiation. The unlabeled dotted line is the joint linear model for Mallard nests in 1976.

models, which included linear and quadratic effects of $\operatorname{AGE}\left(A\right.$ and $A^{2}$ ), linear and quadratic effects of DATE ( $D$ and $D^{2}$ ), and the interaction between AGE and DATE $\left(A^{*} D\right)$. We also fitted joint linear models in AGE $\left(A_{1}+A_{2}\right)$, which involved two straight lines that intersect at the 11-15-day AGE category (e.g. Fig. 1). The models were judged by comparing Mean Square Error (MSE) among them and using the significance level of each effect included in the model.

Table 4 summarizes the results of fitting eight models to each of the four data sets. Bluewinged Teal data for 1976 show a pronounced effect due to the age of nests. This effect is linear, because the models including a quadratic term or joint linear terms do not yield a smaller MSE. Mortality rates seem to depend also on the DATE; the $A+D$ model has a somewhat lower MSE than the $A$ model, although the DATE effect is only marginally significant $(P=0.06)$. Adding the $A^{*} D$ interaction term reduces the MSE slightly but nonsignificantly $(P=0.24)$. The most appropriate model for 1976 Blue-winged Teal data thus includes the linear effects of AGE and DATE. The coefficient for AGE is -0.00102 , which suggests that the daily mortality rate decreased about 0.10 percentage points for each day a nest ages. The coefficient for DATE is 0.000453 , which implies that daily mortality rate increased during the season about 0.45 percentage points for each 10-day period.

Mallard data for 1976 also exhibit a strong effect due to the age of nests. This AGE effect is more nonlinear than for Blue-winged Teal nests; the effect due to the addition of $A^{2}$ in the model is significant at $P=0.09$. The joint linear model has a smaller MSE, however, suggesting that the nonlinearity is not quadratic. The addition of DATE to this model further reduces the MSE, but not significantly $(P=$ 0.11). For 1976 Mallards, the most appropriate model includes the two linear terms in AGE. The coefficient of AGE for nests less than 1115 days old is -0.004408 ; the coefficient for nests at least $11-15$ days old is -0.000137 . These coefficients suggest that the mortality rate of Mallard nests in 1976 declined about 0.44 percentage points/day for the first 10 days and 0.01 percentage points/day thereafter. The rate of decline for the older nests is not significantly different from zero $(P=0.80)$.

Blue-winged Teal data clearly demonstrate declining daily mortality rates with AGE in 1977. This decline is nonlinear. No effect due to DATE is evident. The best-fitting model includes two linear terms in AGE, with coefficients -0.003955 for nests less than 11-15 days old and -0.000130 for older nests. Thus, the 


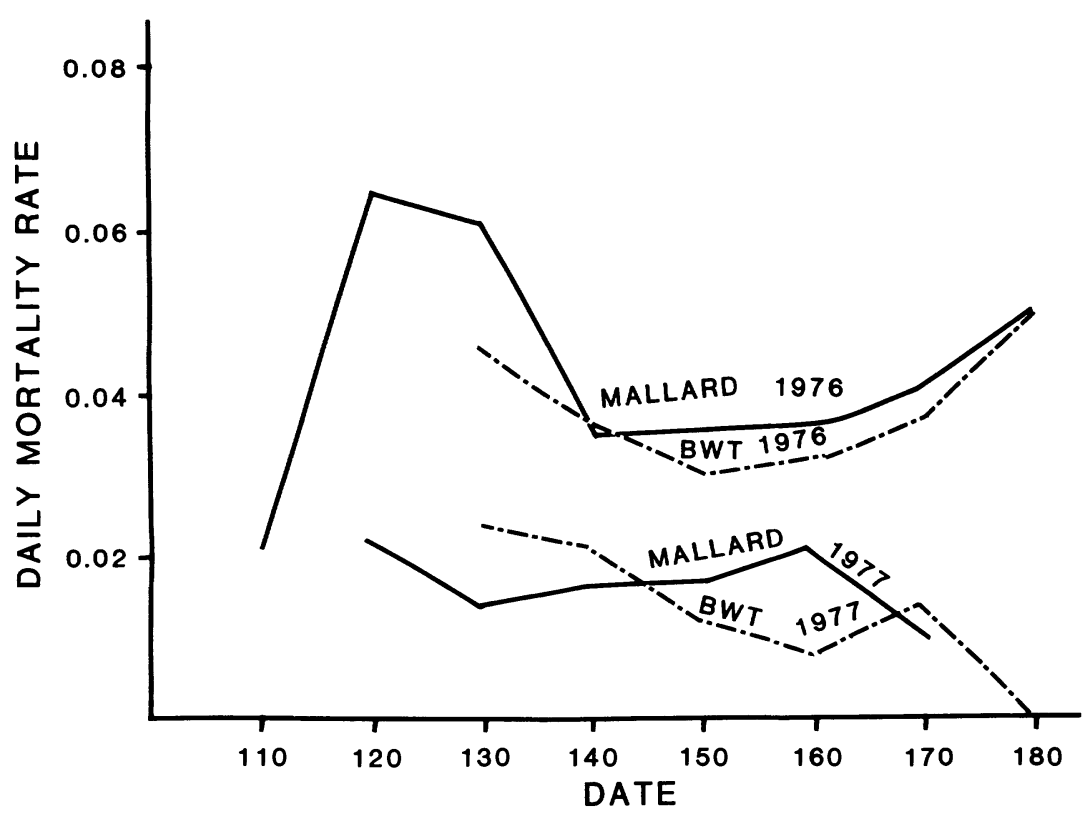

Fig. 2 Daily mortality of nests in relation to 10 -day calendar period.

mortality rate declined about 0.40 percentage points/day for the first 10 days and 0.01 percentage points/day thereafter. The decline among older nests is again nonsignificant $(P=$ 0.62 ).

Mallard data also show a nonlinear decline in mortality rates with AGE in 1977. Again, no DATE effect is detected. The joint linear model fits the data very well. Coefficients of -0.003842 and -0.000753 indicate that the daily mortality rate of 1977 Mallard nests declined 0.38 percentage points/day for the first 10 days and 0.08 percentage points/day thereafter. These coefficients are similar to the values for Bluewinged Teal nests in 1977. The decline among older nests is significant at $P=0.07$.

Hatch rates calculated as the product of age class survival.-When differences occur in mortality rates among nests, hatch rates calculated by the Mayfield Method will be biased high if nests with the highest risk to loss are not proportionately represented in the sample. For example, in our data it appears that nests in the early laying period are at greater risk than those in other age classes. The nests in the 15-day age class are underrepresented in our samples, because nest attendance rates are low and mortality rates are high during this stage. In contrast, nearly all nests in the oldest cohort are discovered, because they were present during four or five searches.

To reduce the above-mentioned bias, May- field (1961, 1975) and Johnson (1979) suggested calculating hatch rates as the product of ageclass survival rates (referred to as the Product Method in this paper) whenever significant age-related differences in mortality can be recognized. We illustrate how hatch rates can be estimated in the presence of age-related variability by referring to the 1976 Blue-winged Teal data. In order to hatch, a nest must survive 5 days at the 1-5-day rate, 5 days at the $6-10$ day rate, and so on, including 4 days at the 3134-day rate. The probability of hatch is thus, from the bottom of Table 3,

$$
\begin{aligned}
(1-0.0472)^{5}(1-0.392)^{5} \ldots & \\
(1-0.302)^{4} & =0.2950 .
\end{aligned}
$$

When we treated our data in this way, we observed a decline in the estimated hatch rates for all data sets, but especially in the two 1977 samples (Table 1). This could be expected, because the Product Method is very sensitive to comparatively low estimated survival rates in any age class. Differences in survival rates between age classes are least variable in the 1976 teal set (Fig. 1, Table 3), and the difference in hatch rates calculated by the Mayfield and Product Methods is only 1.2 percentage points. Relatively low estimated survival in the 1-5day age class in the other data sets results in hatch-rate estimates $3.4-12.8$ percentage points lower than the Mayfield rates. 
TABLE 4. Mean square error (MSE) resulting from fitting eight models to each data set. $A$ and $D$ represent linear effects of age and date, respectively; $A^{2}$ and $D^{2}$ the corresponding quadratic effects; $A^{*} D$ the interaction; and $A_{1}$ and $A_{2}$ linear effects of age for nests $<$ and $\geqslant 11-15$ days. Italicized terms indicate models that provided best fit to data on basis of MSE and significance of included terms.

\begin{tabular}{lccccc}
\hline \hline & \multicolumn{2}{c}{1976} & & \multicolumn{2}{c}{1977} \\
\cline { 2 - 3 } Model & Blue-winged Teal & Mallard & & Blue-winged Teal & Mallard \\
\hline$A$ & 0.01946 & 0.02100 & & 0.00747 & 0.01054 \\
$A+A^{2}$ & 0.01982 & 0.02030 & & 0.00641 & 0.00986 \\
$A_{1}+A_{2}$ & 0.01997 & 0.01961 & & 0.00565 & 0.00975 \\
$A+D$ & 0.01813 & 0.02067 & & 0.00755 & 0.01034 \\
$A+D+A^{*} D$ & 0.01793 & 0.02100 & & 0.00729 & 0.01044 \\
$A+A^{2}+D$ & 0.01847 & 0.01987 & & 0.00647 & 0.00965 \\
$A_{1}+A_{2}+D$ & 0.01862 & 0.01909 & & 0.00566 & 0.00958 \\
$A+A^{2}+D+D^{2}$ & 0.01871 & 0.02020 & & 0.00659 & 0.00987 \\
\hline
\end{tabular}

Hatch rates calculated by the Intercept Method.- Johnson (1979) presented a method for determining heterogeneity among nests in the situation in which no age-related variation was present. The method involved a linear regression of daily mortality rate against the age of nest at discovery. The rationale for the method is that nests found at early ages would include all subpopulations in approximate proportion to their relative abundance, while nests found at older ages would be increasingly biased toward high-survival subpopulations. The data in the last line of Table 3 can be used to illustrate the method with our 1976 Blue-winged Teal sample. A linear regression of these daily mortality rates against the midpoint of the age intervals yields a slope of -0.00062 and an intercept of 0.0463 . The intercept is an estimate of the rate corrected for sampling bias. Raising the value to the 34 th power gives an estimated hatch rate for teal in 1976 of only $20.0 \%$ (Table 1), compared to $30.7 \%$ by the Mayfield Method.

We believe that the discrepancy between these two estimates is at least partially due to the age-related decline in daily mortality rate, which was demonstrated earlier. Even a completely homogeneous population of nests, if it embodies a change in daily mortality rate with age of nest, will exhibit the features of heterogeneity noted by Johnson (1979). Careful interpretation is necessary to determine the actual cause.

\section{Discussion}

Causes of variability in daily mortality rates.In our study we detected a decline in daily mortality rates as the age of the nests increased. In three of the four samples, this decline was sharpest during the first 10 days. As an explanation of these results, we propose: the presence of the female at the nest site, differences in vulnerability to predators among nest sites, or both. The amount of time spent on nests appears to have an inverse relationship to daily mortality rates, which suggests that the presence of the hen is a deterrent to some egg predators. Hens spend increasingly more time at the nest site as laying progresses and, except for occasional daytime recesses, are present day and night during incubation. Caldwell and Cornwell (1975) reported that the average time Mallard hens spend on the nest each day increased from $1.2 \mathrm{~h}$ at egg 3 to 15.2 $h$ at egg 10. Similar behavior was reported by McKinney (1967) and Afton (1979) for Northern Shoveler (A. clypeata) and by Gates (1958) for Gadwall ( $A$. strepera). We assume that Bluewinged Teal behave similarly.

The distracting behavior of hens flushed by predators might serve to reduce egg predation. Many hens defecate on their eggs when disturbed; Swennen (1968) presents evidence that this has a strong repellant effect on predators. Girard (1941) observed Mallard hens defending their nest against crows (Corvus brachyrhynchos) and once saw a hen chase a Columbian ground squirrel (Citellus columbianus) away from her nest. Milne (1974) suggested that the European Eider's (Somateria mollissima) habit of remaining at the nest, even during the laying stage, may have developed from the need to protect eggs against such predators as crows and gulls. Avian predators were not a factor in nest losses during our study, but Franklin's ground squirrels appeared to be fairly common, and $35 \%$ of the nests found had a history of missing eggs, which suggested ground squirrel predation.

Observed differences in daily mortality might be related to the differences in vulnerability of nest sites to predation (Ricklefs 1969: 
6). Nests at high-risk sites are quickly found and destroyed by predators, while attrition at safer sites proceeds at a slower pace. For example, there is some evidence that the vulnerability of nests on our study area was related to the location of nests between the highway pavement and the right-of-way boundary fence (Oetting 1970, Klett, unpubl. data). We suspect that the traffic flow repels some predators, resulting in high survival rates near the highway, while the boundary fence and associated tow-vehicle track may serve as a travel lane for nest predators.

There are rather strong a priori reasons for suspecting that daily mortality rates might vary with calendar periods. As the nesting season progresses, the physical condition of hens deteriorates, the vegetation in which nests are placed increases in height and density, some predators of eggs change behavior patterns as a result of the birth and development of their young, and alternative foods for the predators (buffer prey) become increasingly available. The net result of all these changes was detectable in only the largest of our four data sets. We think that DATE effects were probably real but of less total magnitude and importance than AGE effects.

Appropriate methodology.-The method most applicable to a nesting study depends on the assumptions that the data satisfy. As mentioned earlier, the Traditional Method is proper if only those nests found on the day of initiation are used. This approach is usually impractical for studying species that conceal their nests, as most nests are found some days after initiation and cannot be used. Some investigators use the Traditional Method to estimate hatch rates of nests found after termination. This procedure is acceptable if the chance of finding these nests is independent of their fate, a condition that is not met if some nests are lost before a recognizable nest structure is built or if nests disheveled by predators are more likely to be found than successful ones (Kalmbach 1938).

The Mayfield Method is strictly appropriate only when the daily mortality rate is constant, that is, it does not vary by age of nest, calendar period, nest location, or any other feature. Estimates will not be misleading, however, if the variability is mild. This robustness is fortunate, because many nest samples are too small to be tested for variations in daily mortality, and the Mayfield Method is then the most appropriate.

If daily mortality rates vary with some recognizable feature, such as habitat or calendar period, the appropriate technique is a stratified Mayfield estimator. Simply stated, the Mayfield Method is applied separately to each recognizable group, and a weighted average formed. For example, suppose nests occur in two habitats. In the first habitat, the daily mortality rate is 0.05 ; in the second it is 0.10 . If it is known that nests are twice as common in the first habitat and the period that nests must survive is 10 days, then the weighted Mayfield estimate of the hatch rate is as follows:

$$
2 / 3(1-0.05)^{10}+1 / 3(1-0.10)^{10}=0.515 .
$$

If daily mortality rate varies by age, then we recommend the Product Method, which is simply the Mayfield Method applied within homogeneous periods. The Product Method does require adequate samples of nests within each age class to ensure proper estimates. This requirement is often difficult to achieve in field studies, particularly for nests of early ages, which, in our study at least, were most vulnerable to predators.

Three of our data sets exhibit a joint linear form with high and decreasing age-related mortality early and, thereafter, low and constant mortality. The fourth data set is better fit by another model but does not differ greatly from the joint linear form. Our method of distributing nest losses and exposure days, within 5-day AGE-DATE categories (Appendix), may have contributed to this linear form of decline in daily mortality rates. The decline might appear curvilinear, as suggested by Klimstra and Roseberry (1975), if it had been possible to calculate rates on a daily basis. The latter authors, who attempted to find all the Bobwhite (Colinus virginianus) nests on their study areas, reported daily mortality rates in the laying stage as 0.0564 for $0-3$ eggs and 0.0316 for 4 or more eggs. The rate for nests in incubation was 0.0207. Reed (1975) used the Mayfield Method to estimate daily losses of Black Duck ( $A$. rubripes) nests: 0.07 in the laying period, 0.03 in the first half of the incubation period, and 0.018 in the second half of the incubation period.

A fairly consistent pattern of age-dependen- 
cy in daily mortality rates might emerge if a large number of nesting studies were conducted with adequate samples. Such a pattern, if fairly widespread, could provide a basis for more accurate estimates, even with limited data from young nests.

Another possible situation involves pure heterogeneity. The daily mortality rate for each nest is constant throughout the lifespan, but nests differ amongst themselves; the Intercept Method (Johnson 1979) is applicable in such a situation. We doubt, however, that pure heterogeneity commonly exists; it is more likely combined with and disguised by age-related variability. Further, it has been shown not to be of serious consequence unless differences among groups are large and the proportion of nests at higher risk is large (Johnson 1979). The Mayfield Method, and particularly the Product version, seems to account reasonably well for heterogeneity.

\section{RECOMMENDATIONS}

The choice of nesting-study methodology will depend on the habitats, species reproductive characteristics, study objectives, and economic constraints. The species of concern in this study can be characterized as singlebrooded, ground-nesting birds with a long nesting season, large clutches, and precocial young. Nest-survival rates are often low, but the species are indeterminate layers with the capability of renesting when early clutches are unsuccessful. Recommendations that follow are for species with similar nesting habits.

To meet the objective of most studies, nest searches should be made at regular intervals throughout the nesting season to assure that mortality that is related to nest age, seasonal effects, and other influences is adequately sampled. The cable-chain drag is an efficient tool for obtaining large samples of nests on our study area but is of limited value for obtaining adequate samples of nests in the early laying stage.

When the objective is to estimate the hatch rate of all nests initiated with a minimum of bias, efforts should be made to find as many nests as possible in the early stage of laying. Searches should be made approximately weekly throughout the nesting season, especially during those hours when most of the laying occurs. The sample should be large enough to justify an analysis of variance test for AGE and
DATE effects; we suggest a sample goal of 100 or more nests. We would use the Mayfield Method if daily mortality is constant or variation is moderate; the Product Method is appropriate if significant age-related variation is present. Variation related to seasonal or habitat differences can be handled by some form of stratification. Studies are often designed to compare hatch rates between different years, habitat types, or treatments. To meet these objectives, we recommend a minimum of 50 nests in each unit to be compared. Searches should be made at 2- or 3-week intervals. These minimum samples would most likely restrict the investigator to the Mayfield Method for evaluation purposes.

\section{ACKNOWLEDGMENTS}

We thank the North Dakota State Highway Department for the opportunity to conduct this study on the I-94 right-of-way; Douglas L. Pieske and William J. Schaller for assistance during the 1976 field season; Leo M. Kirsch and other staff of the Northern Prairie Wildlife Research Center for assistance during the 1977 field season; Kenneth F. Higgins, Harold F. Duebbert, Harvey W. Miller, and Alan B. Sargeant for helpful comments on the manuscript; and Lewis M. Cowardin for numerous consultations and editorial assistance. We also thank the former Directorate of the Northern Prairie Wildlife Research Center, W. Reid Goforth and David L. Trauger, for their encouragement and support.

\section{Literature Cited}

Afton, A. D. 1979. Time budget of breeding Northern Shovelers. Wilson Bull. 91: 42-49.

Barr, A. J., J. H. Goodnight, J. P. SAll, \& J. T. Helwig. 1976. A user's guide to SAS 76. Raleigh, North Carolina, SAS Institute.

Caldwell, P. J., \& G. W. Cornwell. 1975. Incubation behavior and temperatures of the Mallard duck. Auk 92: 706-731.

Coulson, J. C. 1956. Mortality and egg production of the Meadow Pipit with special reference to altitude. Bird Study 3: 119-132.

Gates, J. M. 1958. A study of the breeding behavior of the Gadwall in northern Utah. Unpublished M.S. thesis. Logan, Utah, Utah State Univ.

Girard, G. L. 1941. The Mallard: its management in western Montana. J. Wildl. Mgmt. 5: 233-259.

Hammond, M. C., \& W. R. Forward. 1956. Experiments on causes of duck nest predation. J. Wildl. Mgmt. 20: 243-247.

Higgins, K. F., L. M. Kirsch, \& I. J. BALL, JR. 1969. A cable-chain device for locating duck nests. J. Wildl. Mgmt. 33: 1009-1011.

JoHnson, D. H. 1979. Estimating nest success: the 
Mayfield method and an alternative. Auk 96: 651-661.

KalmbaCH, E. R. 1938. A comparative study of nesting waterfowl on the Lower Souris Refuge: 1936-1937. Trans. North Amer. Wildl. Conf. 3: 610-623.

Kilmstra, W. D., \& J. L. Roseberry. 1975. Nesting ecology of the Bobwhite in southern Illinois. Wildl. Monogr. No. 41.

LACK, D. 1954. The natural regulation of animal numbers. Oxford, Clarendon Press.

Mayfield, H. 1961. Nesting success calculated from exposure. Wilson Bull. 73: 255-261.

- 1975. Suggestions for calculating nest success. Wilson Bull. 87: 456-466.

MCKInNEY, F. 1967. Breeding behaviour of captive Shovelers. Wildfowl 18: 108-121.

MilleR, H. W., \& D. H. Johnson. 1978. Interpreting the results of nesting studies. J. Wildl. Mgmt. 42: 471-476.

Milne, H. 1974. Breeding numbers and reproductive rate of Eiders at the Sands of Forvie National Nature Reserve, Scotland. Ibis 116: 135-152.

Oetring, R. B. 1970. Waterfowl nesting on interstate highway right-of-way in North Dakota. Unpublished M.S. thesis. Fargo, North Dakota, North Dakota State Univ.

Peakall, D. B. 1960. Nest records of the Yellowhammer. Bird Study 7: 94-102.

REED, A. 1975. Reproductive output of Black Ducks in the St. Lawrence Estuary. J. Wildl. Mgmt. 39: 243-255.

RiCKLEFS, R. E. 1969. An analysis of nesting mortality in birds. Smithsonian Contrib. Zool. 9: 148.

Stewart, R. E., \& H. A. Kantrud. 1971. Classification of natural ponds and lakes in the glaciated prairie region. U.S. Bur. Sport Fish. Wildl. Resources Publ. 92.

SWENNEN, C. 1968. Nest protection of Eiderducks and Shovelers by means of faeces. Ardea 56: 248-258.

Voorhees, L. D., \& J. F. CAssel. 1980. Highway right-of-way: mowing versus succession as related to duck nesting. J. Wildl. Mgmt. 44: 155163.

Weller, M. W. 1956. A simple field candler for waterfowl eggs. J. Wildl. Mgmt. 20: 111-113.

\section{Appendix}

\section{Method Used to Calculate Mortality Rates by Age Class and Calendar Period}

We calculated daily mortality rates for nests in each 5-day period of age (1-5 days, 6-10 days, etc.) and for each 10-day calendar period (Days 110-119, 120129 , etc.). To do so, we calculated the exposure of all nests in each category and the number of such nests destroyed. The daily mortality rate is the number destroyed divided by the number of nest-days exposure. As an example, consider a nest found at age 3 days (laying with 3 eggs) on Day 118 (28 April). It was checked on Day 125 (5 May) and found to be still viable. Thus, there were 0 destructions occurring during 7 nest-days of exposure. The exposure was distributed over the AGE-DATE categories as follows:

\begin{tabular}{rrrrrrrr}
\hline AGE: & 3 & 4 & 5 & 6 & 7 & 8 & 9 \\
DATE: & 118 & 119 & 120 & 121 & 122 & 123 & 124 \\
Exposure: & 1 & 1 & 1 & 1 & 1 & 1 & 1 \\
Losses: & 0 & 0 & 0 & 0 & 0 & 0 & 0
\end{tabular}

Hence the following AGE-DATE categories would receive the indicated contributions to exposure:

\begin{tabular}{ccc}
\hline & \multicolumn{2}{c}{ AGE } \\
\cline { 2 - 4 } DATE & $1-5$ & $6-10$ \\
\hline $110-119$ & 2 & 0 \\
$120-129$ & 1 & 4 \\
\hline
\end{tabular}

Had the nest been destroyed by the visit on Day 125 , then one loss would be attributed to the nest and the exposure reduced, because the expectation of the number of days the nest was at risk is less than 7 . The single loss would be distributed over the 7 days in the interval according to the probability that the nest was destroyed on that day. To evaluate that probability, we assumed that nest mortality occurred at a constant rate of $5 \%$ /day. Then the probability that a nest was destroyed, for example, by the next day (Day 119), if it was destroyed when next visited on Day 125 , is

$$
\begin{aligned}
& \operatorname{Pr}\{\text { survive exactly } 0 \text { days } \mid \text { not survive } 7 \text { days }\} \\
& \quad=\frac{\operatorname{Pr}\{\text { survive exactly } 0 \text { days }\}}{1-\operatorname{Pr}\{\text { survive at least } 7 \text { days }\}} \\
& =\frac{(0.95)^{0}(1-0.95)}{1-(0.95)^{7}} \\
& =0.1657 .
\end{aligned}
$$

The distribution of the single loss over the 7-day period between visits to the nest is as shown in the following table:

\begin{tabular}{crrrrrrrr}
\hline AGE: & 3 & 4 & 5 & 6 & 7 & 8 & 9 \\
DATE: & 118 & 119 & 120 & 121 & 122 & 123 & 124 \\
Exposure: & 1.0000 & 0.8343 & 0.6768 & 0.5272 & 0.3851 & 0.2501 & 0.1218 \\
Losses: & 0.1657 & 0.1575 & 0.1496 & 0.1421 & 0.1350 & 0.1282 & 0.1218 \\
\hline
\end{tabular}

The exposure is also distributed over the interval. The exposure for Day 118 will be 1 , because the nest was exposed to risk on that day. The exposure accruing to Day 119 will be 
E\{exposure on Day 119|not survive to Day 125 \} $=1 \times \operatorname{Pr}\{$ survive at least 1 day $\mid$ not survive 7 days $\}$

$=\sum_{j=1}^{6} \operatorname{Pr}\{$ survive exactly $j$ days $\mid$ not survive 7 days $\}$ $=\sum_{j=1}^{6} \frac{(0.95)^{j}(1-0.95)}{1-0.95^{7}}$

$=\frac{(0.95)^{1}\left(1-0.95^{7-1}\right)}{1-0.95^{7}}$

$=0.8343$.

The distribution of exposure over the 7 days between visits is as shown in the preceding table. From that table we can determine the contribution of the single nest to the losses and exposure for AGE-DATE categories. The following table shows losses/exposure for each category.

\begin{tabular}{ccc}
\hline & \multicolumn{2}{c}{ AGE } \\
\cline { 2 - 3 } DATE & $1-5$ & $6-10$ \\
\hline $110-119$ & $0.3232 / 1.8343$ & $0 / 0$ \\
$120-129$ & $0.1496 / 0.6768$ & $0.5271 / 1.2842$
\end{tabular}

After adding nest losses and exposure in each category, daily mortality was calculated by the Mayfield Method. 